\title{
Separation of Polyhydroxyalkanoates-Producing Bacterial Strains Using PHA Synthase Gene and Their Evaluation for PHA Deposition
}

\author{
Abdul Basit Khan ${ }^{1 *}$, Mehmood Iqbal Khattak ${ }^{2}$, Omer Mukhtar Tarar ${ }^{1}$, Farzana Habib ${ }^{2}$, \\ Khalid Jamil $^{1}$, Arfa Yasmin ${ }^{2}$ and Shoukat Parvez ${ }^{3}$ \\ ${ }^{1}$ Food and Marine Resources Research Centre; PCSIR Laboratories Complex; Karachi; Pakistan. ${ }^{2}$ Applied \\ Chemistry Research Centre; PCSIR Laboratories Complex; Karachi; Pakistan. ${ }^{3}$ Head Office; PCSIR Laboratories \\ Complex; Islamabad
}

\begin{abstract}
In this study, a variety of samples were screened for the presence of PHA synthase gene. Results showed that 16 out of 102 isolated were positive for PHA respective genes. The highest prevalence was observed in Pseudomonas aeruginosa. The capability of PHA production was also shown by growing these strains on the defined medium and subsequent analysis using intracellular granules staining and Fourier transform infrared spectroscopy (FT-IR). The microscopic analysis showed that the positive strains accumulated PHA in the cell. The FT-IR analysis showed the presence of PHA peaks in the dried cells as well as in extraction product. P aeruginosa strain P7 showed higher concentration of PHA compared to the others as demonstrated by the highest respective peaks in FT-IR.
\end{abstract}

Key words: Polyhydroxyalkanoates, Biodegradable polymers, PHA synthase gene, FTIR

\section{INTRODUCTION}

The accumulation of petrochemical polymers in the surroundings and growing awareness about the environmental pollution throughout the world has triggered the search for new products that are compatible with the environment. Currently, most polymer products are designed and prepared synthetically and very limited consideration is paid for their ultimate disposal. Of particular concern are throw-away plastic products, which are very suitable to use due to their physical properties. They have replaced wood, glass and other packaging materials since their emergence in 1940s (Ojumu 2004). However, these nondegradable plastics are building up in the environment at the rate of 25 million tons per year, which may persist for hundreds of years (Voaides 2010). It is worthy under these circumstances to design and develop biodegradable materials, which ensures an appropriate disposal with reference to the environment and eco system. In this regard, materials such as polyhydroxyalkanoates (PHAs), polylactic acid and others environment-friendly biopolymers are especially useful.

Polyhydroxyalkanoates (PHA) are a class of naturally occurring polyesters that are produced and accumulated as inclusion bodies of carbon and energy reserve material in different microorganisms (Jendrossek 2009). It has been estimated that over 300 bacterial species and more than 90 genera, including Gram-negative and Gram-positive organisms accumulate various

*Author for correspondence: basit_24@yahoo.com 
PHAs (Kim 2007; Razzaq 2010). These polyesters are generally synthesized in two forms depending on the conditions and strains. Short-chain length hydroxyalkanoic acids (SCL-PHAs) with 3-5 carbon side chains and medium-chain length PHAs (MCL-PHAs) having carbon ranging from 6-14 atoms (Kim 2007; Jendrossek 2009). In addition, numbers of copolyesters carrying these SCL-PHAs and MCL-PHAs have also been reported (Lutke-Eversloh 2001; Steinbuchel 2003). There is a considerable attraction in PHAs due to their biodegradability and thermoplastic properties. However, the high cost of production compared to cheap petrochemical polymers, prevents their use on industrial scale. Continuous efforts are being made and several studies are going on to develop a cost-effective strategy by using inexpensive substrates as a carbon source and high yield of biopolymers (Cromwick 1996; Verlinden 2011).

The aim of this study was to screen various samples of different origin for PHA producing bacterial strains using PCR for PHA synthase gene and other conventional methods. Subsequently, positive microorganisms were evaluated for PHA accumulation on defined medium.

Table 1 - Samples Description.

\begin{tabular}{clcclc}
\hline S. No. & Type of samples & Number of isolates & S. No. & Type of samples & Number of isolates \\
\hline 1 & Garden Soil & 13 & 8 & Lens Solution (Washing) & 08 \\
2 & Domestic Waste water & 17 & 9 & Milk Products & 03 \\
3 & Air (Laboratory) & 15 & 10 & Spinach & 01 \\
4 & Tap Water & 11 & 11 & Meat & 05 \\
5 & Rice & 11 & 12 & Fish meal & 06 \\
6 & Wheat & 05 & 13 & Drinking water & 03 \\
7 & Spices & 04 & & Total & $\mathbf{1 0 2}$ \\
\hline
\end{tabular}

Type of samples tested with number of bacterial strains isolated from these samples.

\section{Polymerase Chain Reaction}

To carry out the PCR experiment, a single colony was picked into molecular biology grade water and heated to $95^{\circ} \mathrm{C}$ for 10 minutes. All the isolates were screened for phaCl and phaC2 genes using I-179L and I-179R primers and PCR conditions as reported in the literature (Solaiman 2000; Jamil 2007). The sequences of the primers were;

Forward: I-179L: 5'-ACAGATCAACAAGTTC TACATCTTCGAC-3',

Reverse: I-179R: 5'-GGTGTTGTCGTTGTTCC AGTAGAGGATGTC-3'.

Briefly, $2.5 \mu$ bacterial suspension was added to $12.5 \mu 1$ of GoTaq ${ }^{\circledR}$ Green Master mix (Promega),

\section{MATERIALS AND METHODS}

\section{Samples Collection and Isolation of Bacterial Strains}

A total of 35 samples of different origin were collected to isolate the bacterial strains for PHA production, and 102 isolates were included in this study (Table 1). Garden Soil, waste water, tap water and drinking water samples were collected from different part of Karachi; air samples were collected by plate exposure method. These samples were subjected to total aerobic count by pour plate method, except air samples isolates, which were directly obtained through the plate exposure method in different parts of the laboratory premises. Briefly, the samples were homogenized in Butterfield phosphate buffer (stock solution: $34 \mathrm{~g}$ of $\mathrm{KH}_{2} \mathrm{PO}_{4}$ dissolved in $1000 \mathrm{ml}$ of distilled water, $\mathrm{pH} 7.2 \pm 0.5$; working solution: $1.25 \mathrm{ml}$ of stock solution $/ 1000 \mathrm{ml}$ of distilled water). Serial dilutions, i.e. 1:10 to 1:10000 were prepared and $1.0 \mathrm{ml}$ of sample from all dilutions was inoculated on Petri plates. Molten Plate Count Agar was added, allowed to solidify and incubated at $35^{\circ} \mathrm{C}$ for $48 \mathrm{~h}$. The bacterial isolates were picked on the basis of their colonial morphology and streaked on Tryptic Soy Agar for pure culture.
$0.5 \mu \mathrm{L}$ of each primer and $9.0 \mu \mathrm{L}$ of molecular biology grade water. All the PCR tubes were placed in Biorad thermal cycler and PCR was started by initial denaturation at $94^{\circ} \mathrm{C}$ for 5 minutes, followed by 30 cycles of denaturation at $94^{\circ} \mathrm{C}$ for 1 minute, annealing at $50^{\circ} \mathrm{C}$ for 2 minutes, and extension at $72^{\circ} \mathrm{C}$ for 2 minutes. The thermal cycling was terminated with a final extension of $72^{\circ} \mathrm{C}$ for 5 minutes. Agarose gel electrophoresis was performed using $1 \%$ agarose and ethidium bromide staining to analyse the PCR products. Gels were exposed to UV light and photograph was taken after electrophoresis. 


\section{Identification of PHA Positive Strains}

All the bacterial isolates positive for phaCl and phaC2 genes were subjected to Gram staining and subsequent identification. RapID identification strips (remel) were used to identify these strains. Bacterial strains were sub-cultured to obtain a single colony and preceded according to manufacturer's instruction. Results of RapID strips were interpreted and reports were generated using online Eric web software of remel.

\section{PHA Production}

Experiments were carried out to confirm the production of PHA by the strains positive for respective genes. For the evaluation of PHA, the isolates were grown in $10 \mathrm{ml}$ of Tryptic Soy Broth (TSB) at $35^{\circ} \mathrm{C}$ for $24 \mathrm{~h}$. Cells were harvested by centrifugation and re-inoculated in medium "E" prepared as described earlier with slight modification (Cromwick 1996). The medium "E" was prepared by adding $\mathrm{K}_{2} \mathrm{HPO}_{4}(5.8 \mathrm{~g} / \mathrm{l}), \mathrm{KH}_{2} \mathrm{PO}_{4}$ $(3.7 \mathrm{~g} / \mathrm{l}), \quad \mathrm{MgSO}_{4} .7 \mathrm{H}_{2} \mathrm{O} \quad(0.25 \mathrm{~g} / \mathrm{l}), \quad\left(\mathrm{NH}_{4}\right)_{2} \mathrm{SO}_{4}$ $(0.03 \mathrm{~g} / \mathrm{l})$, and $1.0 \mathrm{ml}$ of micronutrient solution prepared in $1 \mathrm{M} \mathrm{HCl}$ having $\mathrm{FeSO}_{4} .7 \mathrm{H}_{2} \mathrm{O}(2.78 \mathrm{~g} / \mathrm{l})$, $\mathrm{MnCl}_{2} .4 \mathrm{H}_{2} \mathrm{O} \quad(1.98 \mathrm{~g} / \mathrm{l}), \mathrm{CoSO}_{4} .7 \mathrm{H}_{2} \mathrm{O} \quad(2.81 \mathrm{~g} / \mathrm{l})$, $\mathrm{CuCl}_{2} \cdot 2 \mathrm{H}_{2} \mathrm{O}(0.17 \mathrm{~g} / \mathrm{l}), \mathrm{ZnSO}_{4} \cdot 7 \mathrm{H}_{2} \mathrm{O}(0.29 \mathrm{~g} / \mathrm{l})$, and $\mathrm{CaCl}_{2} \cdot 2 \mathrm{H}_{2} \mathrm{O}(1.67 \mathrm{~g} / \mathrm{l})$. Glucose $(2 \%)$ was used as a sole source of carbon. The flasks were incubated at $30^{\circ} \mathrm{C}$ and $150 \mathrm{rpm}$ shaking for $48 \mathrm{~h}$ after inoculation.

\section{Staining for PHA Granules}

Intracellular PHA granules were observed by staining with Sudan black-B as reported earlier (Santhanam 2010). After incubation, heat-fixed smears were prepared and dipped in $0.3 \%$ Sudan Black-B prepared in ethylene glycol for 20 minutes. Slides were then decolorized in xylol and after drying, counter-stained with safranin solution for 30 seconds. Slides were washed and observed under the microscope.

\section{Extraction and Characterization}

Incubated culture medium was centrifuged at 5000 rpm; cells were harvested and washed with deionized water to extract the PHA. Cell pellets were dried at $100^{\circ} \mathrm{C}$ in the oven. Dried cell mass was initially checked for characteristic PHA peaks by FT-IR (Thermo Nicolet Avatar 320 spectrophotometer USA) using ATR technique. Subsequently, dried cell mass was used for PHA extraction by refluxing in $100 \mathrm{ml}$ chloroform for
$4 \mathrm{~h}$ as described earlier (Annuar 2008). The chloroform extract was slowly added to stirred cold methanol to precipitate the PHA, which was re-dissolved in chloroform and precipitated again in cold methanol. Precipitate was then separated and air-dried for characterization.

\section{RESULTS AND DISCUSSION}

Polyhydroxyalkanoates (PHA) is an expression comprising a family of polyesters produced by a diverse group of microorganisms. Variety of intracellular storage compounds, including glycogen, polyamino acids, polyphosphates, etc. have been identified in the microbes during the excess availability of nutrients, and PHA is one of them (Sudesh and Doi 2005). The polymerization of PHA in the cells is mainly dependent on an enzyme, called PHA synthase, which catalyzes the reaction to produce $\mathrm{PHA}$ and free $\mathrm{CoA}$ from hydroxyacyl-coenzyme A (Rehm 2004). As stated earlier, more than 300 bacterial species are able to accumulate the PHA, but the deposition in most of them are very low (Nobia 2007). The use of mixed cultures are gaining interest and it has been concluded in many reports that mixed microbial cultures could be more favorable for PHA production compared to pure cultures (Dias 2008; Tian 2009). In addition, recombinant organisms having pha gene expressions also proved to be a good candidate for mass scale PHA production (Nikel 2006).

In this study, 102 bacterial stains were randomly selected from 35 samples of different origin. PCR was performed to screen these strains for phaC1/C2 genes. It was observed that 16 strains were positive for respective genes and amplified characteristic $\sim 540$ bp PCR products (Fig. 1). The size of the product was in agreement with the studies reported earlier (Solaiman 2000; Jamil 2007). Table 2 shows the affiliation of these strains with their respective samples. Strains positive for the respective genes were identified on the basis of their Gram reaction and biochemical characteristics. All the positive strains were Gram negative, non-lactose fermentor rods. RapID identification strips NF plus (remel) were utilized to observe their biochemical characters and their identity. It was observed that 12 out of 16 positive strains belonged to $P$. aeruginosa species (Table 2). 


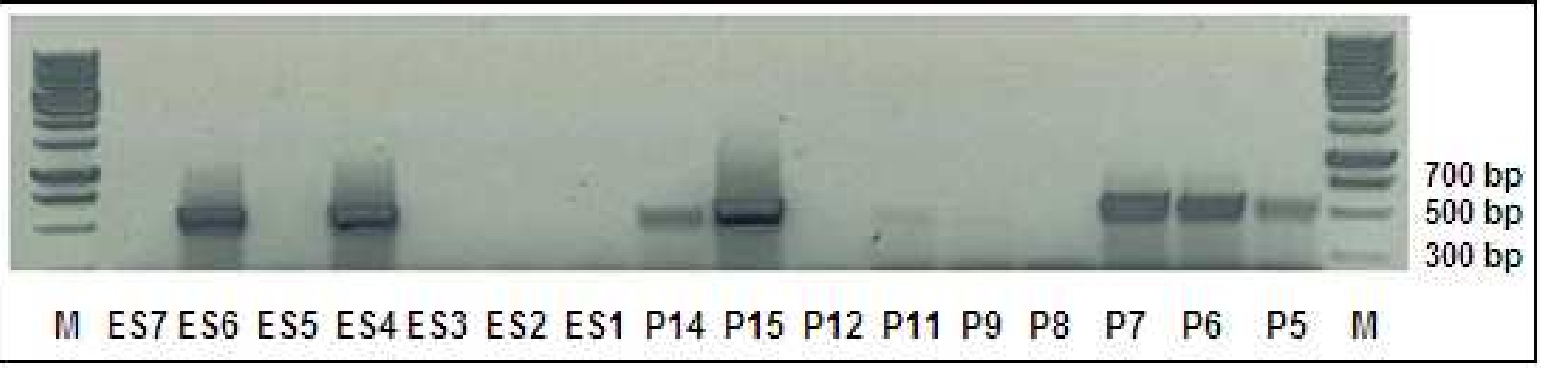

Figure 1 - Polymerase Chain Reaction for phaC1/C2 genes. PCR products of $\sim 540$ bp were obtained in PHA positive strains with primers I-179L and I-179R. M = Molecular weight marker $(1 \mathrm{~kb}$, Bioron $)$. Bacterial Strains $=$ ES7-P5.

Table 2 - Identification of PHA positive strains using RapID NF plus identification strips (remel).

\begin{tabular}{ccccc}
\hline S No & ID & Source & Gram Reaction & Organism Identified \\
\hline 1. & ES4 & Air & G-ve, rods, scattered & Comamonas acidovorans \\
2. & ES6 & Air & G-ve, rods, scattered & Pseudomonas aeruginosa \\
3. & TR11 & Tap Water & G-ve, rods, scattered & Pseudomonas aeruginosa \\
4. & TR13 & Tap Water & G-ve, rods, scattered & Pseudomonas aeruginosa \\
5. & TR21 & Waste water & G-ve, rods, scattered & Alcaligenes faecalis \\
6. & P1 & Lens Solution (W) & G-ve, rods, scattered & Pseudomonas aeruginosa \\
7. & P3 & Lens Solution (W) & G-ve, rods, scattered & Pseudomonas aeruginosa \\
8. & P4 & Lens Solution (W) & G-ve, rods, scattered & Pseudomonas aeruginosa \\
9. & P5 & Lens Solution (W) & G-ve, rods, scattered & Pseudomonas aeruginosa \\
10. & P6 & Lens Solution (W) & G-ve, rods, scattered & Pseudomonas aeruginosa \\
11. & P7 & Lens Solution (W) & G-ve, rods, scattered & Pseudomonas aeruginosa \\
12. & P13 & Meat (Beef) & G-ve, rods, scattered & Pseudomonas aeruginosa \\
13. & P14 & Soil & G-ve, rods, scattered & Pseudomonas aeruginosa \\
14. & P15 & Tap water & G-ve, rods, scattered & Pseudomonas aeruginosa \\
15. & TR49 & Tap Water & G-ve, rods, scattered & Sphingomonas paucimobilis \\
16. & TR50 & Tap Water & G-ve, rods, scattered & Stenotrophomonas maltophila \\
\hline
\end{tabular}

PHA production has been widely investigated in Gram-negative species such as members of Ralstonia, Alkaligenes, Pseudomonas, and recombinant $E$ coli. In this study, it was demonstrated that phaCl/phaC2 was commonly distributed among the Gram-negative bacterial isolates from different sources, including air, water, soil, and lens solution washings. Investigation for this gene revealed that around $16 \%$ carried PHA synthase gene with the highest prevalence in $P$. aeruginosa. In another study, around $40 \%$ of strains isolated from 20 soil samples were positive for PHA production (Chen 2000). This significant difference could be due to the analysis of variety of samples. Fluorescent
Pseudomonads including $P$. aeruginosa have been extensively studied and reported to accumulate large amounts of PHAs as carbon storage compounds (Huisman 1989; Pham 2004). They are also probably the most versatile in terms of broad substrate specificity (Sudesh and Doi 2005). All positive strains for respective genes were further screened to determine the intracellular deposition of PHA. Organisms were grown on medium "E" containing glucose as a carbon source. The microscopic examination of the cultures after Sudan black showed that all strains positive for PHA synthase genotype accumulated PHA polymers in the cell (Fig. 2). 


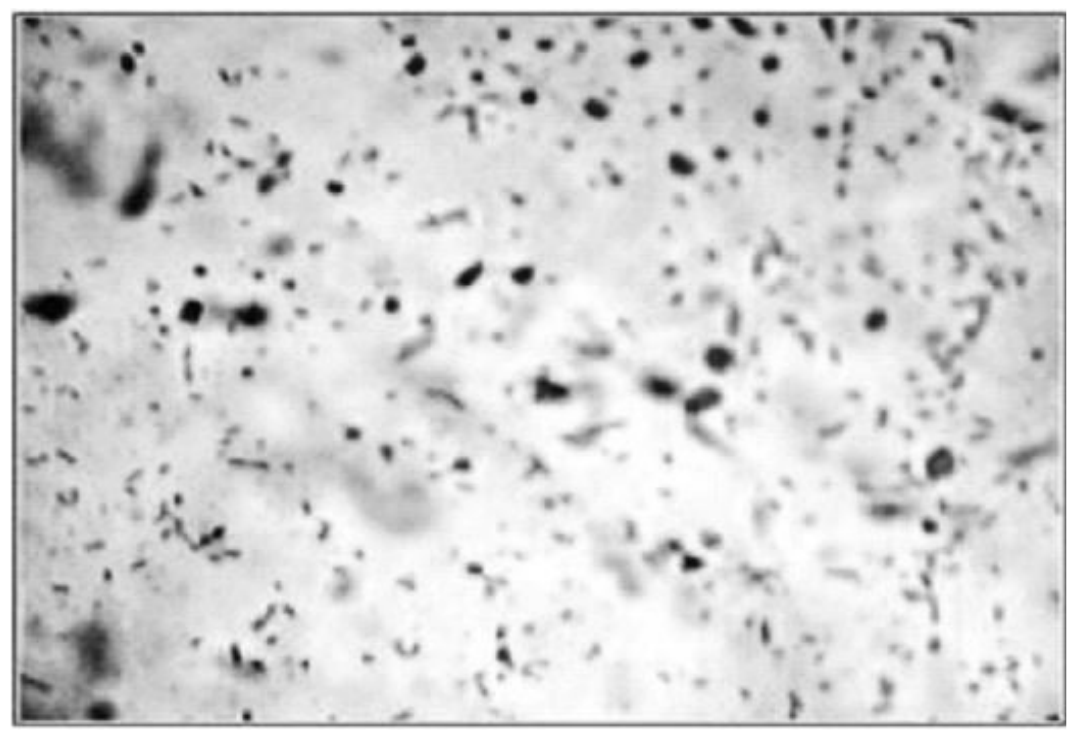

Figure 2 - Staining of PHA. Photograph of stained PHA organism. Intracellular PHA granules are in black and rest is in light colour.

In addition, FT-IR spectroscopy was employed to detect the functional group of PHA in the dried cell mass before and after the extraction. Solvent extraction using chloroform and methanol was performed and extracts were tested using FT-IR technique. PHA extraction can also be done by aqueous phase using sodium hypochlorite, which is comparatively a cheaper method, but it reduces the molecular weight of the polymer (Luzier 1992). A FT-IR using ATR technique was used in this study with the spectra range of $4000-500 \mathrm{~cm}^{-1}$. Several methods have been reported for the qualitative analysis of PHA, including GC, NMR, etc., but FT-IR is reported to be the most rapid and simple method (Hong 1999). It does not require complicated sample preparation and can be used to detect the extracted PHA as well as intracellular PHA in the dried cells (Hong 1999). It was observed that all the strains positive for phaC1/phaC2 genes also showed PHA in the dried cells before and after the extraction (Figures 3,4). Strains showed strong peaks ranging from 1728 to $1744 \mathrm{~cm}^{-1}$ which were attributed to the stretching vibration of ester carbonyl group $\mathrm{C}=\mathrm{O}$ peak (Figures 3, 4). In addition, strong peaks were also observed at 2922, 2923 and $2925 \mathrm{~cm}^{-1}$, which represented the stretching vibration of $\mathrm{C}-\mathrm{H}$ methylene group. Similar pattern of peaks in FTIR analysis were observed in other studies when glucose containing medium was used for PHA production (Nisha 2012). Although other esters may also generate a similar pattern, but no such interruption was observed when phaCl/phaC2 negative strains were tested for PHA (data not shown). Moreover, peak shifts were observed when intact cells FT-IR were compared with the FT-IR purified product. It has been reported earlier, which was mainly due to the complex chemical environment in the dried cell mass (Hong 1999).

Out of 16 positive strains, $P$. aeruginosa $\mathrm{P} 7$ strain showed the highest peaks, both in the dried cells and in the extract, and this intensity of PHA region peaks roughly showed the comparatively higher PHA concentration in the respective strain. 

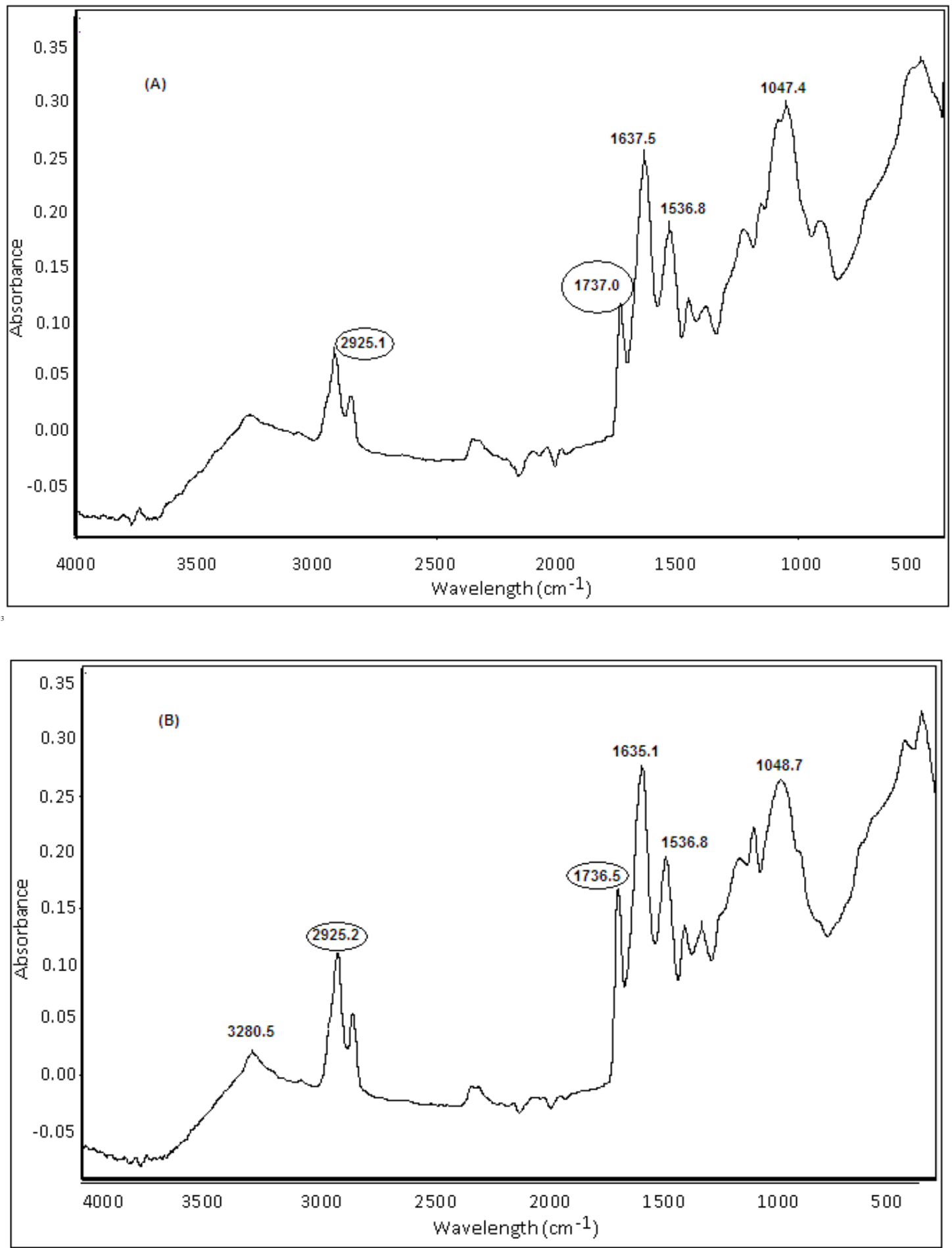

Figure 3 - Fourier-transform infrared (FT-IR) spectra of dried cell mass before extraction of (A) P6 (B) P7. 

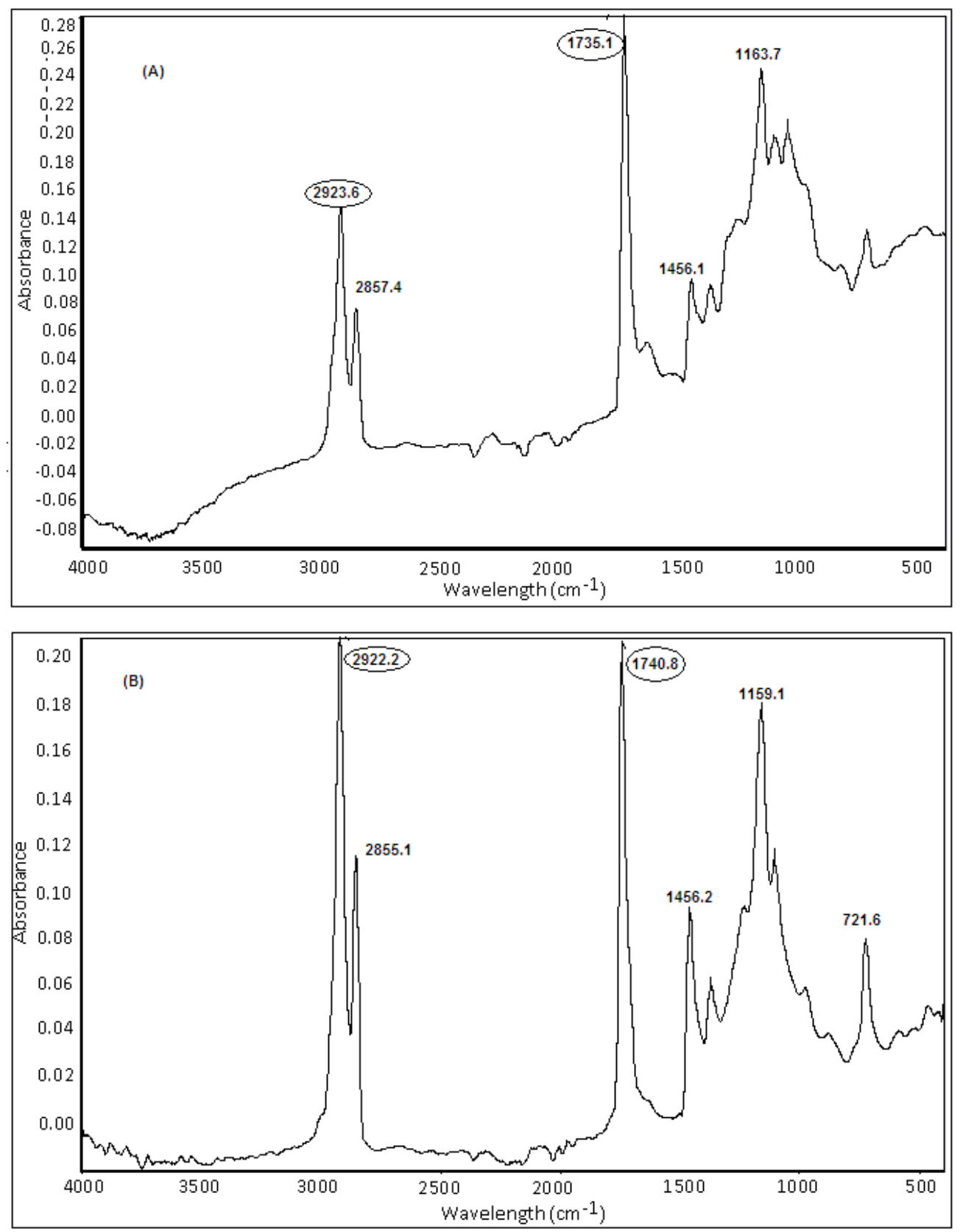

Figure 4 - Fourier-transform infrared (FT-IR) spectra of dried cell mass after extraction of (A) P6 (B) P7.

\section{CONCLUSION}

The objective of this study was to see the prevalence of PHA producing organisms in different samples through PCR and their potential to produce the intracellular PHA. It was observed that the genes for PHA synthase were usually distributed in Gram-negative organisms. It was also shown that the rapid screening for PHA producers could be done in the dried cells before and after the extraction through FT-IR.

\section{REFERENCES}

Annuar MSM, Tan IKP, Ramchandran KB. Evaluation of nitrogen sources for growth and production of medium-chain-length poly-(3-hydroxyalkanoates) from palm kernel oil by Pseudomonas putida PGA1. AsPac J Mol Biol Biotechnol. 2008; 16(1): 11-15. 
Chen G-Q, Wu Q, Zhao K, Yu PH. Functional polyhydroxyalkanoates synthesized by microorganisms. Chinese J Polym Sci. 2000; 18(5): 389-396.

Cromwick A-M, Foglia T, Lenz RW. The microbial production of poly(hydroxyalkanoates) from tallow. Appl Microbiol Biotechnol. 1996; 46: 464-469.

Dias JML, Oehmen A, Serafim L, Lemos P, Reis M, Oliveira R. Metabolic modeling of polyhydroxyalkanoate copolymers production by mixed microbial cultures. BMC Syst Biol. 2008; 2:59.

Hong K, Sun S, Tian W, Chen GQ, Huang W. A rapid method for detecting bacterial polyhydroxyalkanoates in intact cell by Fourier transform infrared spectroscopy. Appl Microrobiol Biotechnol. 1999; 51: 523-526.

Huisman GW, De Leeuv O, Eggink G, Witholt B. Synthesis of poly 3-hydroxyalkanoates is a common feature of fluorescent Pseudomonads. Appl Environ Microbiol. 1989; 55(8): 1949-1954.

Jamil N, Ahmed N, Edwards DH. Characterization of biopolymer produced by Pseudomonas sp. CMG607w of marine origin. J Gen Appl Microbiol. 2007; 53: 105-109.

Jendrossek D. Polyhydroxyalkanoate granules are complex subcellular organelles (Carbonosomes). J Bacteriol. 2009; 191(10): 3195-3202.

Kim DY, Kim HW, Chung MG, Rhee YH. Biosynthesis, modification, and biodegradation of bacterial medium-chain-length polyhydroxyalkanoates. J Microbiol. 2007; 45(2): 8797.

Lutke-Eversloh T, Bergander K, Luftmann $\mathrm{H}$, Steinbuchel A. Identification of a new class of biopolymer: Bacterial synthesis of a sulfur-containing polymer with thioester linkages. Microbiol. 2001; 147: 11-19.

Luzier WD. Materials derived from biomass/biodegradable materials. Proc Natl Acad Sci USA. 1992; 89: 839-842.

Nikel PI, Almeida AD, Melillo EC, Galvagno MA, Pettinari MJ. New recombinant Escherichia coli strain tailored for the production of poly (3hydroxybutarate) from agroindustrial by-products. Appl Environ Microbiol. 2006; 72(6): 3949-3954.

Nisha J, Mudaliar N, Senthilkuman P, Narendrakumar, Samrot AV. Influence of substrate concentration in accumulation pattern of poly $(\mathrm{R})$ hydroxyalkanoate in Pseudomonas putida SU-8. Afr J Microbiol Res. 2012; 6(15):3623-3630.

Nobia M, Ivonne G, Dionisio M, Victoria G, Dolly R, Diego S, et al. Bioprospecting and characterization of poly- $\beta$-hydroxyalkanoate (PHAs) producing bacteria isolated from Colombian sugarcane producing areas. Afr J Biotechnol. 2007; 6(13): 1536-1543.
Ojuma TV, Yu J, Solomom BO. Production of polyhydroxyalkanoates, a bacterial biodegradable polymer. Afr J Biotechnol. 2004; 3(1): 18-24.

Pham TH, Webb JS, Rehm BHA. The role of polyhydroxyalkanoate biosynthesis by Pseudomonas aeruginosa in rhamnolipid and alginate production as well as stress tolerance and biofilm formation. Microbiol. 2004; 150: 3405-3413.

Razzaq A, Jamil N, Naheed N, Hasnain S. Bacteria from contaminated urban and hilly areas as a source of polyhydroxyalkanoates production. Afr $J$ Biotechnol. 2010; 9(13): 1919-1925.

Rehm BHA, Steinbuchel A. PHA synthases: The key enzymes for PHA synthesis. In: Steinbuchel A. Editor. Biopolymers. Wiley-VCH; 2004. pp. 173-180.

Sanathanam A, Sasidharan S. Microbial production of polyhydroxyalkanoates (PHA) from Alcaligens spp. And Pseudomonas oleovorans using different carbon sources. Afr J Biotechnol. 2010; 9(21): 3144-3150.

Solaiman DKY, Ashby RD, Foglia TA. Rapid and specific identification of medium-chain-length polyhydroxyalkanoate synthase gene by polymerase chain reaction. Appl Microbiol Biotechnol. 2000; 53: 690-694.

Steinbuchel A, Lutke-Eversloh T. Metabolic engineering and pathway construction for biotechnological production of relevant polyhydroxyalkanoates in microorganisms. Biochem Eng J. 2003; 16: 81-96.

Sudesh K and Doi Y. Polyhydroxyalkanoates. In: Catia Bastioli Editor. Handbook of Biodegradable polymers. Rapra Technology Limited; 2005. pp. 219256.

Tian P-Y, Shang L, Ren H, Mi Y, Fan D-D, Jiang M. Biosynthesis of polyhydroxyalkanoates: current research and development. Afr J Biotechnol. 2009; 8(5): 709-714.

Verlinden RAJ, Hill DJ, Kenward MA, Williams CD, Piotrowska-Seget Z, Radecka IK. Production of polyhydroxyalkanoates from waste frying oil by Cupriavidus necator. AMB Express. 2011; 1:11.

Voaides C, Groposila D, Ciuca M, Lupescu I, Pop A, Cornea CP. PHAs accumulation in Pseudomonas putida P5 (wild type and mutant) in lipid containing media. Rom Biotech Lett. 2010; 15(4): 5467-5473.

Received: September 30, 2012; Accepted: May 21, 2013. 